\title{
A Tool for Automatic Correction of Endogenous Concentrations: Application to BHB Analysis by LC-MS-MS and GC-MS
}

\author{
Brigitte Desharnais ${ }^{\mathrm{a}, \mathrm{b}, *}$, Marie-Jo Lajoie ${ }^{\mathrm{a}}$, Julie Laquerre ${ }^{\mathrm{a}}$, Stéphanie Savard ${ }^{\mathrm{a}}$, Pascal \\ Mireault ${ }^{\mathrm{a}}$, Cameron D. Skinner ${ }^{\mathrm{b}}$ \\ ${ }^{a}$ Department of Toxicology, Laboratoire de sciences judiciaires et de médecine légale \\ 1701 Parthenais Street, Montréal, Québec, Canada H2K 3S7 \\ ${ }^{b}$ Department of Chemistry $\&$ Biochemistry, Concordia University \\ 7141 Sherbrooke Street West, Montréal, Québec, Canada H4B 1 R6
}

\begin{abstract}
Several substances relevant for forensic toxicology purposes have an endogenous presence in biological matrices: beta-hydroxybutyric acid (BHB), gamma-hydroxybutyric acid (GHB), steroids and human insulin, to name only a few. The presence of significant amounts of these endogenous substances in the biological matrix used to prepare calibration standards and quality control samples (QCs) can compromise validation steps and quantitative analyses. Several approaches to overcome this problem have been suggested, including using an analog matrix or analyte, relying entirely on standard addition analyses for these analytes, or simply ignoring the endogenous contribution provided that it is small enough. Although these approaches side-step the issue of endogenous analyte presence in spiked matrix-matched samples, they create serious problems with regards to the accuracy of the analyses or production capacity. We present here a solution that addresses head-on the problem of endogenous concentrations in matrices used for calibration standards and quality control purposes. The endogenous analyte concentration is estimated via a standard-addition type process. This estimated concentration, plus the spiked concentration are then used as the de facto analyte concentration present in the sample. These de facto concentrations are then used in data analysis software (MultiQuant, Mass Hunter, etc.) as the sample's concentration. This yields an accurate quantification of the analyte, free from interference of the endogenous contribution. This de facto correction has been applied in a production setting on two BHB quantification methods (GC-MS and LC-MS-MS), allowing the rectification of BHB biases of up to $30 \mu \mathrm{g} / \mathrm{mL}$. The additional error introduced by this correction procedure is minimal, although the exact amount will be highly method-dependent. The endogenous concentration correction process has been automated with an $\mathrm{R}$ script. The final procedure is therefore highly efficient, only adding four mouse clicks to the data analysis operations.
\end{abstract}

*Author to whom correspondence should be addressed. Email: brigitte.desharnais@msp.gouv.qc.ca 


\section{Introduction}

Almost all bioanalytical method validation procedures involve spiking authentic matrices to evaluate figures of interest, such as precision, accuracy and matrix effects [1]. When endogenous analytes are targeted for quantification, the usual validation protocol can be difficult or impossible to apply. The authentic matrices (e.g., blood, urine or hair) which are typically used to spike known concentrations of analytes cannot be found blank of the targeted substance. Ignoring the endogenous amount, invariably of unknown magnitude, present in these authentic matrices introduces a systematic bias in all analyses. Not only does this pose a problem at the method validation stage, but standard production operations are compromised as well. The accepted practice in bioanalysis methods is to spike calibration standards and quality control samples (QCs) with internal standards in authentic matrices (or a pool of authentic matrices) to control for potential matrix effects. Again in this situation, the endogenous analyte will introduce a bias in the calibration equation, compromising, to various degrees, the quantitative accuracy of the result.

A number of analytes of interest in forensic toxicology are endogenous compounds. A few common examples include gammahydroxybutyrate (GHB), cyanide, carboxyhemoglobin (HbCO), insulin and steroids. Some other analytes, such as alcohol consumption markers or caffeine are not endogenous $[2,3,4]$ but can be highly prevalent in the population $[3,5$ ? ]; thus finding authentic matrices free from them can also be difficult. The case of the endogenous compound beta-hydroxybutyric acid (BHB) is specifically examined here. BHB acts as a diabetic or alcoholic ketoacidosis biomarker, and is a hyperglycemia indicator [6, 7]. Concentrations up to $50 \mu \mathrm{g} / \mathrm{mL}$ in blood are generally considered normal, whereas concentrations from 50 to $200 \mu \mathrm{g} / \mathrm{mL}$ are considered to be elevated $[6,7]$. The decision point with regards to ketoacidosis is generally between 200 and $250 \mu \mathrm{g} / \mathrm{mL}[6,7]$.

Some publications have highlighted the problem posed by these analytes in the method validation and production contexts [8]. A number of possible solutions to this issue have been suggested. A comprehensive, albeit time and resource-consuming option, is to perform quantification of those analytes via standard addition methods $[8,9,10,11]$. While this effectively eliminates the issue of endogenous presence in the spiking matrix, its use in a high-volume production context is not sustainable. The use of a surrogate matrix [8, 12, 13, 14], e.g., synthetic blood, or a surrogate analyte [8, 14, 15, 16], e.g. a stable-isotope labeled version of the targeted analyte can also be considered. In the end, these methods yield a more or less accurate approximation of the figure sought. For example, the accuracy of the target analyte in real blood cannot be assured, since the assumption that the targeted analyte behaves in synthetic blood in the same way as it would in real blood is probably untenable for a wide variety of analytes due to different analyte specific matrix effects [8]. In that sense, while bioanalysts might settle for a surrogate matrix or analyte, it is clearly a less than perfect solution. Finally, it is possible to ignore entirely the endogenous concentration in the spiking matrix, provided that it is small enough and can be dismissed as a low-level interference (e.g. $<10 \%$ of lowest calibration standard) [1,9]. Of course, this approach is quite appealing in its simplicity. The downside is that a bias, however small, will systematically be included 
in all the quantitative analyses performed. Additionally, this process might require the screening of several matrix sources (e.g. blood lots) to find one with an endogenous level low enough to fit the acceptance criteria, using precious time and resources.

In reality, these methods have been designed to side-step the issue of endogenous analytes in spiking matrices, but they each have their significant drawback and don't address the problem head-on. In this paper, we suggest a simple and comprehensive automated solution to correct for the presence of endogenous analytes in spiking matrices.

\section{Materials and methods}

\section{1. $G C-M S$ BHB analysis}

Calibration standards and quality control samples were spiked in ante-mortem human whole blood (Utak, 44600-WB(F), Valencia, CA, USA) at concentrations of 0.00 , 10.0, 20.0, 50.0, 100, 200, 425 and $500 \mu \mathrm{g} / \mathrm{mL}$ (standards) and 60.0, 150 and $375 \mu \mathrm{g} / \mathrm{mL}$ (QCs) using BHB (Sigma-Aldrich, H6501, Saint-Louis, MO, USA).

In a conical tube, $50.0 \mu \mathrm{L}$ of blood and $25.0 \mu \mathrm{L}$ of internal standard solution $(400 \mu \mathrm{g} / \mathrm{mL}$ BHB- $_{4}$ dissolved in methanol, CDN isotopes, D-6088, Pointe-Claire, QC, Canada) were mixed. Protein precipitation was performed by adding $225 \mu L$ of acetonitrile and vortexing immediately. After centrifugation $(9600 \times g, 7 \mathrm{~min}), 100 \mu \mathrm{L}$ of supernatant was evaporated to dryness under nitrogen, reconstituted in $250 \mu \mathrm{L}$ ethanol $(\geq 99.9 \%$, Commercial Alcohols by Greenfield Global, P210EAAN, Brampton, ON, Canada) and reevaporated to dryness under nitrogen. Derivatization was performed for a maximum of 10 vials at a time to prevent evaporation by adding $70.0 \mu \mathrm{L}$ acetonitrile (HPLC Grade, $\geq 99.9 \%$, EMD millipore corporation, AX0156-1, Billerica, MA, USA) and 70.0 $\mu$ L BSTFA+TMCS (99:1) (Cerilliant, B-023, Round Rock, TX, USA) and vortexing. Incubation was tested during method development but showed no significant advantage and was therefore not used $[17,18]$.

After centrifugation (3 $200 \times g, 5 \mathrm{~min}), 1 \mu \mathrm{L}$ of extract was separated on an Agilent HP-5MS column ( $15 \mathrm{~m}, 0.25 \mathrm{~mm}$ i.d., $0.25 \mu \mathrm{m}$ film thickness) using a 16.25 minutes separation. The injector was kept at $280{ }^{\circ} \mathrm{C}$ and operated in pulsed split mode, with a split ratio of 3:1 (MS Agilent MSD 5975 C) or 20:1 (MS Agilent MSD 5977B HES). The GC was operated with a $1 \mathrm{~mL} / \mathrm{min}$ helium flow and an oven program of $70^{\circ} \mathrm{C}$ to $110^{\circ} \mathrm{C}$ over 8 minutes followed by an increase to $280^{\circ} \mathrm{C}$ over 4.25 minutes and a plateau of 4 minutes. Pressure at the head of the column was 1.9 psi. The mass spectrometer parameters were set as follows: mass range of 41 to $400 \mathrm{~m} / \mathrm{z}$, scan rate of $3.95 \mathrm{scan} / \mathrm{min}$, solvent delay of $2.50 \mathrm{~min}$, acquisition time of $16.25 \mathrm{~min}$, ionization source temperature of $230^{\circ} \mathrm{C}$, quadrupole temperature of $150{ }^{\circ} \mathrm{C}$, transfer capillary temperature of $280^{\circ} \mathrm{C}$ and electron impact energy of $70 \mathrm{eV}$. The method was validated on Agilent gas chromatographs 7890 A and 7890B, equipped with Agilent MSD $5975 \mathrm{C}$ or 5977B HES and automated injector 7693. The data acquisition and analysis software used was MassHunter@B.07.04.2260, B.08.00 build 8.0.598.0. 
The method has been validated under SWGTOX [1], ISO 17025:2005 [19] and CAN-P $1578[20]$ standards.

\section{2. $L C-M S-M S B H B$ analysis}

Calibration standards and quality control samples were prepared in ante-mortem human whole blood (Utak, 44600-WB(F), Valencia, CA, USA) at concentrations of 0.00 , $3.00,6.00,30.0,60.0,150,255$ and $300 \mu \mathrm{g} / \mathrm{mL}$ (standards) and 9.0, 120 and $240 \mu \mathrm{g} / \mathrm{mL}$ (QCs) using BHB (Sigma-Aldrich, H6501, Saint-Louis, MO, USA).

In a 96-well plate $(2 \mathrm{~mL}$ square wells, Fisher Scientific, AB-0932, Ottawa, ON, Canada), $100 \mu \mathrm{L}$ of blood and $10.0 \mu \mathrm{L}$ of internal standard solution $\left(330 \mu \mathrm{g} / \mathrm{mL}\right.$ GHB-D $_{6}$ dissolved in methanol, CND isotopes, D-5462, Pointe-Claire, QC, Canada) were mixed. Note that calibration dynamic range and internal standard differ between the GC-MS and LC-MS-MS methods; each of these validated methods were already in use in the laboratory and the differences are attributable to various historical and practical constraints. The samples were diluted by adding $100 \mu \mathrm{L}$ of MeOH:0.2\% formic acid in water (50:50 v:v) solution (methanol: EMD Millipore corporation, MX0486-1, Billerica, MA,USA; formic acid: Fisher Scientific, A117, Fair Lawn, NJ, USA). Proteins were then precipitated by adding $400 \mu L$ of acetone:ACN (30:70 v:v, room temperature) solution (acetone:Fisher Scientific, A949, Fair Lawn, NJ, USA; and acetonitrile:EMD Millipore corporation, AX0156-1, Billerica, MA USA). Samples were thoroughly vortexed after each mixing step. After centrifugation $(3200 \times g, 5 \mathrm{~min}), 20.0 \mu \mathrm{L}$ of supernatant was transferred to a second 96-well plate, diluted with $200 \mu \mathrm{L}$ of $\mathrm{MeOH}: 0.2 \%$ formic acid in water (10:90 v:v) and vortexed. An Agilent HPLC 1200 series, or 1260 Infinity, coupled to a Sciex MS/MS 5500 QTrap was used for a 8.75 minute separation of $5 \mu L$ of the extract on an Agilent Zorbax Eclipse Plus C18 column (2.1 x $100 \mathrm{~mm}, 3.5 \mu \mathrm{m})$ kept at $50^{\circ} \mathrm{C}$. A step/ramp gradient with 2:98 methanol:10 $\mathrm{mM}$ ammonium formate $\mathrm{pH} 3.0$ (mobile phase A) and methanol (mobile phase B) was used. Using a flow of $600 \mu \mathrm{L} / \mathrm{min}$, the percentage of mobile phase A was brought from 100\% (0.0 $\mathrm{min})$, to $85 \%$ (0.3 $\mathrm{min})$, to $80 \%$ (3.0 $\mathrm{min})$, to $40 \%(3.5 \mathrm{~min})$, to $20 \%(6.0 \mathrm{~min})$, to $0 \%(6.5 \mathrm{~min})$ for a $0.7 \mathrm{~min}$ wash followed by a 0.9 min re-equilibration period. BHB retention time under these conditions was $0.95 \mathrm{~min}$. Acquisition was carried out in negative multiple reaction monitoring (MRM) mode, with a quantitative Q1/Q3 transition of 103 Da/59 Da (collision energy of $-18 \mathrm{~V}$ and collision cell exit potential of $-7 \mathrm{~V}$ ) and a qualitative Q1/Q3 transition of $103 \mathrm{Da} / 41 \mathrm{Da}$ (collision energy of $-30 \mathrm{~V}$ and collision cell exit potential of $-10 \mathrm{~V}$ ). Other mass spectrometer parameters were as follows: MRM detection window of $60 \mathrm{sec}$, target scan time of $0.25 \mathrm{sec}$, settling time of $50 \mathrm{msec}$, break of $3.00 \mathrm{msec}$, curtain gas (CUR) of 30.00 , collision gas (CAD) of 10.00 , ion spray voltage (IS) of $-4500 \mathrm{~V}$, source heater temperature (TEM) of $700^{\circ} \mathrm{C}$, ion source nebulizer gas (GS1) of 60.00 , heater gas (GS2) of 65.00. Data was acquired using Analyst $囚 1.6 .2$ build 8489 software and analyzed with Multiquant@3.0.1 (Version 3.0.6256.0).

The method has been validated under SWGTOX [1], ISO 17025:2005 [19] and CAN-P 1578 [20] standards. 


\subsection{Correction for the endogenous concentration}

Several known amounts of analyte were added to distinct aliquots (i.e., a calibration curve in the matrix was prepared) to determine the endogenous concentration of the analyte. This was performed by a two-step process. First, the endogenous analyte concentration in the calibration standards was determined via a standard addition process using the known concentrations that were added to the matrix. Second, this endogenous concentration was then added to the nominal concentrations of the standards and QC samples to generate the de facto analyte concentration present.

If the calibration model selected during method validation [21, 22] was linear, the calibration equation was expressed as:

$$
y=b_{1} x+b_{0}
$$

The endogenous concentration $\left(x_{e}\right)$ of the analyte in the biological matrix is the $\mathrm{x}$ intercept; in this case, it is calculated as

$$
x_{e}=\left|\frac{b_{0}}{b_{1}}\right|
$$

If the calibration model selected was quadratic, i.e.,

$$
y=b_{2} x^{2}+b_{1} x+b_{0}
$$

the endogenous concentration was calculated as

$$
x_{e}=\left|\frac{-b_{1}+\sqrt{b_{1}^{2}-4 b_{2} b_{0}}}{2 b_{2}}\right|
$$

The corrected concentration $\left(x_{c}\right)$, i.e., the de facto concentration to be used for standards and QCs, was calculated by adding the endogenous concentration $\left(x_{e}\right)$ to the spiked concentration $\left(x_{s}\right)$ :

$$
x_{c}=x_{s}+x_{e}
$$

To implement these corrections, and remove the bias from the calibration, the tables of calibration standards' and QCs' expected concentrations (or "actual concentration" in MultiQuant software) were reassigned using $x_{c}$ instead of $x_{s}$. This effectively "shifts" the calibration curve to the right and achieves the desired correction; accurate concentrations will be calculated for unknown samples. 
This correction process has been automated by a script written and run in RStudio (RStudio, Boston, MA, USA). R (programming environment, https ://www.r-project . org/) and RStudio (graphical interface, https://www.rstudio.com/) are free opensource statistical software tools. This automated tool requires that the user copy to the clipboard $(\mathrm{Ctrl}+\mathrm{C})$ a table with the concentrations $\left(x_{s}\right)$ in the first column and the signals (e.g., area ratio) in the second column and run ("source") the script in RStudio. Once the script has run, the corrected concentrations $\left(x_{c}\right)$ are automatically stored in the clipboard, ready to be pasted into the data analysis software of the user. The full correction script and instructions for use (including a link to a user video tutorial) are available in Supplementary Data 1.

\subsection{Calculation of the additional error introduced by the endogenous concentration cor- rection}

The purpose of this endogenous concentration correction is to remove the systematic bias (or deterministic error) from the analysis. However, it is equally important to appreciate that the random error associated with determining the endogenous concentration needs to be considered when assessing the error associated with the samples. The exact magnitude of the error folded in by the correction operation can be computed using the formulas for the standard error of the prediction [23].

Since the spiked concentration $\left(x_{s}\right)$ and the endogenous concentration $\left(x_{e}\right)$ are both calculated from the same data set, their errors are dependent on one another. In this situation, the summation of errors should be used for the corrected concentration $\left(x_{c}\right)[24]$ :

$$
s_{x_{c}}=s_{x_{s}}+s_{x_{e}}
$$

rather than the standard error propagation formula $\sqrt{s_{a}^{2}+s_{b}^{2}}$, which is reserved for addition of independent errors [24].

Calculation of the components $s_{x_{s}}$ and $s_{x_{e}}$ will differ depending on whether the data studied is homoscedastic or heteroscedastic.

For homoscedastic data, those items are calculated as

$$
\begin{gathered}
s_{x_{e}}=\frac{s_{e}}{b_{1}} \sqrt{\frac{1}{n}+\frac{\bar{y}^{2}}{b_{1}^{2} \sum\left(x_{i}-\bar{x}\right)^{2}}} \\
s_{x_{s}}=\frac{s_{e}}{b_{1}} \sqrt{\frac{1}{m}+\frac{1}{n}+\frac{\left(\overline{y_{s}}-\bar{y}\right)^{2}}{b_{1}^{2} \sum\left(x_{i}-\bar{x}\right)^{2}}}
\end{gathered}
$$


with

$$
s_{e}=\sqrt{\frac{\sum\left(y_{i}-\hat{y}_{i}\right)^{2}}{n-2}}
$$

Where

$s_{x_{e}}$ is the error on the estimated endogenous analyte concentration;

$s_{x_{s}}$ is the error on the estimated uncorrected analyte concentration;

$s_{e}$ is the square root of the residual variance, representing the spread of the measurements around the fitted regression line (variance of $\mathrm{y}$ given $\mathrm{x}$, often called the error of the regression $S_{r}$ );

$b_{1}$ is the estimated slope of the linear regression;

$n$ is the number of calibration levels (calibration standards);

$m$ is the number of measurement replicates of the analyzed sample;

$\bar{y}$ is the average measurement for all calibration standards;

$\overline{y_{s}}$ is the average measurement for the analyzed sample;

$y_{i}$ is the measurement for the calibration standard at concentration level $x_{i}$;

$\hat{y}_{i}$ is the predicted measurement at concentration level $x_{i}$ (obtained by inserting the value of $x_{i}$ in the calibration equation);

$x_{i}$ is the concentration at level $i$;

$\bar{x}$ is the average concentration for all calibration standards (including blanks).

For heteroscedastic data, the components $s_{x_{s}}$ and $s_{x_{e}}$ are calculated as

$$
\begin{gathered}
s_{x_{e}}=\frac{s_{e}}{b_{1}} \sqrt{\frac{1}{\sum w_{i}}+\frac{\bar{y}_{w}^{2}}{b_{1}{ }^{2}\left(\sum w_{i} x_{i}{ }^{2}-\sum w_{i} \bar{x}_{w}^{2}\right)}} \\
s_{x_{s}}=\frac{s_{e}}{b_{1}} \sqrt{\frac{1}{w_{s} m}+\frac{1}{\sum w_{i}}+\frac{\left(\bar{y}_{s}-\bar{y}_{w}\right)^{2} \sum w_{i}}{b_{1}^{2}\left(\sum w_{i} \sum w_{i} x_{i}^{2}-\left(\sum w_{i} x_{i}\right)^{2}\right)}}
\end{gathered}
$$

with

$$
\begin{gathered}
s_{e}=\sqrt{\frac{\sum w_{i}\left(y_{i}-\hat{y}_{i}\right)^{2}}{n-2}} \\
\bar{y}_{w}=\frac{\sum w_{i} y_{i}}{\sum w_{i}} \\
\bar{x}_{w}=\frac{\sum w_{i} x_{i}}{\sum w_{i}}
\end{gathered}
$$

Where

$w_{i}$ is the weighting factor applied to concentration level $i$;

$w_{s}$ is the weighting factor applied to the sample measurement (concentration level $s$ ); 
$\bar{x}_{w}$ is the weighted mean concentration;

$\bar{y}_{w}$ is the weighted mean measurement;

$s_{x_{e}}, s_{x_{s}}, s_{e}, b_{1}, n, m, \bar{y}, \overline{y_{s}}, y_{i}, \hat{y}_{i}$ and $x_{i}$ are as described above.

Once again, these calculations have been automated via an RStudio script. The user is required to build a table with a "Name", "Type", "Spiked_Conc" and "Measure" headers that store the type of sample ("QC" or "Cal"), the spiked concentration and the measure (e.g., area or area/response ratio). This table is copied to the clipboard $(\mathrm{Ctrl}+\mathrm{C})$, the script is run, and the estimated error for each QC level is displayed in the console. The full error evaluation script and instructions for use (including a link to a user video guide) are available in Supplementary Data 2.

\section{Results and Discussion}

\subsection{Correction of the endogenous concentration}

With "well-behaved" analytes in a matrix devoid of endogenous content, calibration curves should produce a y-intercept $\left(b_{0}\right)$ approximately equal to zero (within the limit of the experimental error). On the other hand, as shown in Figure 1a, a calibration curve for an analyte with a detectable endogenous concentration will show an upward shift. This shift is generated by the constant signal produced, throughout the entire calibration range, by the endogenous concentration. The observed signal is then just the sum of the endogenous signal and the one generated by the analyte spiked into the matrix. Calibration curves with endogenous analytes will therefore present a y-intercept $\left(b_{0}\right)$ significantly different from zero. In order to correct for the systematic bias introduced by endogenous content in the matrix, its contribution can be either ignored or corrected for.

Conceptually, the simplest route is to set the $b_{0}$ parameter in the calibration equation to zero, i.e., to ignore $b_{0}$ entirely (note that this is not the same thing as forcing the intercept through zero, which would modify the $b_{1}$ parameter). Another equivalent way to achieve this correction would be to subtract $b_{0}$, or the average signal obtained for the $0 \mu \mathrm{g} / \mathrm{mL}$ standard(s), from all calibration standards and QCs. These solutions are all adequate, and some software (e.g., Sciex OS, Mass Hunter's Blank Subtraction Add-In) offer tools to carry one of them out. However, the inflexibility of several data analysis software in routine use might render general application of these solutions difficult. If this procedure is to be applied in highthroughput production settings, the best solution will be the one which operates seamlessly within the confines of the data analysis software.

The most practical procedure to meet those requirements involves modifying the expected ("actual") concentration provided to the data analysis software. Since the expected concentration is one of the few adjustable parameters in the analysis tables, this must be used as the tool to effect the correction. As stated in equation [? ](5), the corrected concentration for standards and QCs is one reflecting the real, or de facto, analyte concentration $\left(x_{c}\right)$ in the sample: what was spiked $\left(x_{s}\right)$ plus the amount which was already present to begin with, the endogenous concentration $\left(x_{e}\right)$. Using the real, corrected concentrations to describe calibration standards and QCs will shift the calibration curve (Figure 1b), achieving the desired correction. Once the de facto concentration 


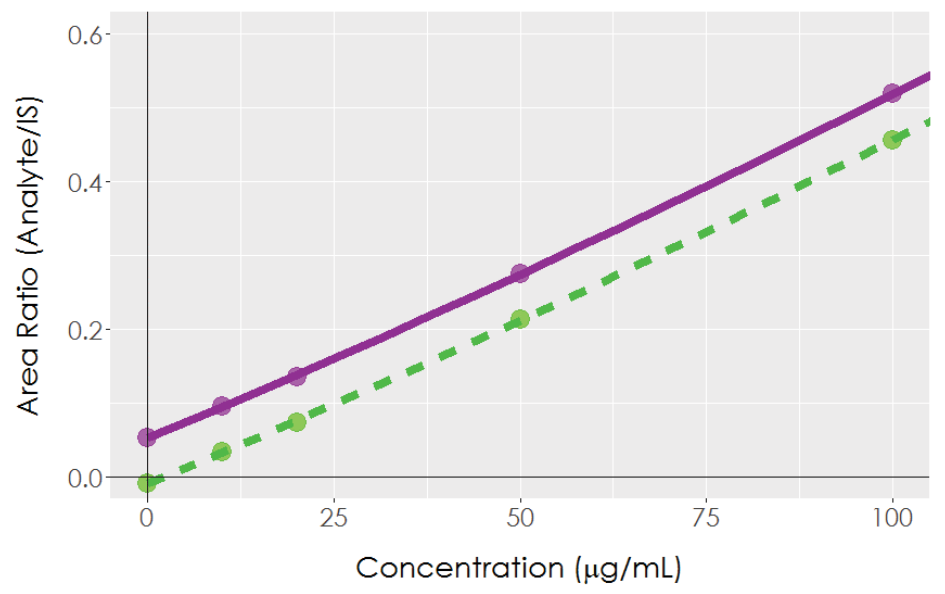

(a) Dashed line: calibration curve for a regular analyte, with no endogenous contribution: the $\mathrm{y}$ intercept $b_{0} \approx 0$. Solid line: calibration curve for an analyte with an endogenous concentration: the y intercept $b_{0}>0$

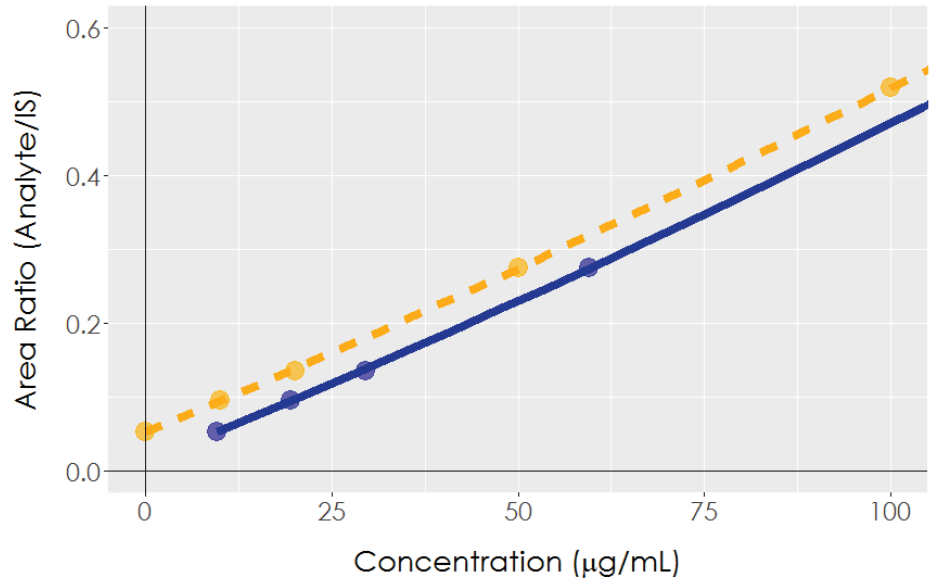

(b) Dashed line: uncorrected calibration curve for an endogenous analyte. Solid line: calibration curve for the endogenous analyte following the correction process using the de facto concentration in the matrix.

Figure 1: Calibration curves in different settings. 
of analyte is used to build the calibration curve, it can perform bias-free quantification of any casework and QC samples.

The correction process can be used in conjunction with either linear or quadratic calibration. To demonstrate this, 10000 calibration data sets (heteroscedastic, $1 / x^{2}$ ) were generated using the range of $b_{0}, b_{1}$ and $b_{2}$ values observed over 14 LC-MS-MS experimental calibration curves with various known levels of "endogenous" analyte. The endogenous concentration was back-calculated using a quadratic, $1 / x^{2}$ regression or using a linear, $1 / \mathrm{x}^{2}$ regression from which the top two standards $(255$ and $300 \mu \mathrm{g} / \mathrm{mL}$ ) were removed to satisfy linear calibration requirements [21,22]. The bias in the calculated endogenous concentration (vs. the known endogenous concentration) was calculated for every data set in both the linear and quadratic regression cases. The histograms of the resulting differences shows that the distribution is more symmetrical, with a median closer to zero when the quadratic regression is used rather than the linear one. The complete results, including the $\mathrm{R}$ script used to perform this in silico evaluation, are available in Supplementary Data 3. Based on these results, the authors recommend using the calibration model selected and validated during the validation process to perform the correction for the endogenous concentration, whether it is quadratic or linear, unweighted, $1 / x$ or $1 / x^{2}$ weighted.

In order to achieve seamless integration into production work, an R Script automating this correction process has been developed and is available in Supplementary Data 1 , along with a user video tutorial. This script is easily configured to fit the needs of the user. Its use in a production setting adds four mouse clicks to the entire analysis process, taking less than 30 seconds to perform.

\section{2. $B H B$ and $G H B$ endogenous concentrations estimated in a production setting}

In our production operations, calibration standards and QCs have all been spiked in authentic human blood matrix and corrected for the presence of endogenous content using the R script available in Supplementary Data 1. Since these methods have been put in production, 17 GC-MS and 83 LC-MS-MS batches have been analyzed, using 2 and 10 different blood lots, respectively. In the GC-MS batches, the estimated endogenous BHB concentrations ranged from 1.1 to $13 \mu \mathrm{g} / \mathrm{mL}$, with a median of $7.5 \mu \mathrm{g} / \mathrm{mL}$. In the LC-MS-MS batches, the estimated BHB concentrations ranged from 0.080 to $30 \mu \mathrm{g} / \mathrm{mL}$, with a median of $4.1 \mu \mathrm{g} / \mathrm{mL}$. A box plot representation of the results per matrix lot is shown in Figure 2. This shows that results are generally coherent within a single matrix lot, despite the occasional outlier. These remote results might even create distortions in the boxplot; for example, lot B appears different in the GC-MS and LC-MS-MS analyses despite similar compositions and medians of 1.5 and $1.18 \mu \mathrm{g} / \mathrm{mL}$ respectively. Extreme values could be attributable to analyte instability or neoformation during storage [25]. Indeed, the same matrix lot has been used for analysis for up to 169 days, potentially long enough to observe degradation. Within the scope of this research, we were unable to ascertain the exact mechanism generating these extreme values. An analysis of five separate GC-MS calibrations, prepared in the same matrix lot, extracted and analyzed in a close timeframe gave coherent endogenous concentrations $\left(x_{e}\right)$. Estimated endogenous concentrations $\left(x_{e} \pm s_{x_{e}}\right)$ were $11.32 \pm 0.14,11.87 \pm 0.18,11.64 \pm 0.17,11.76 \pm 0.16$ and 


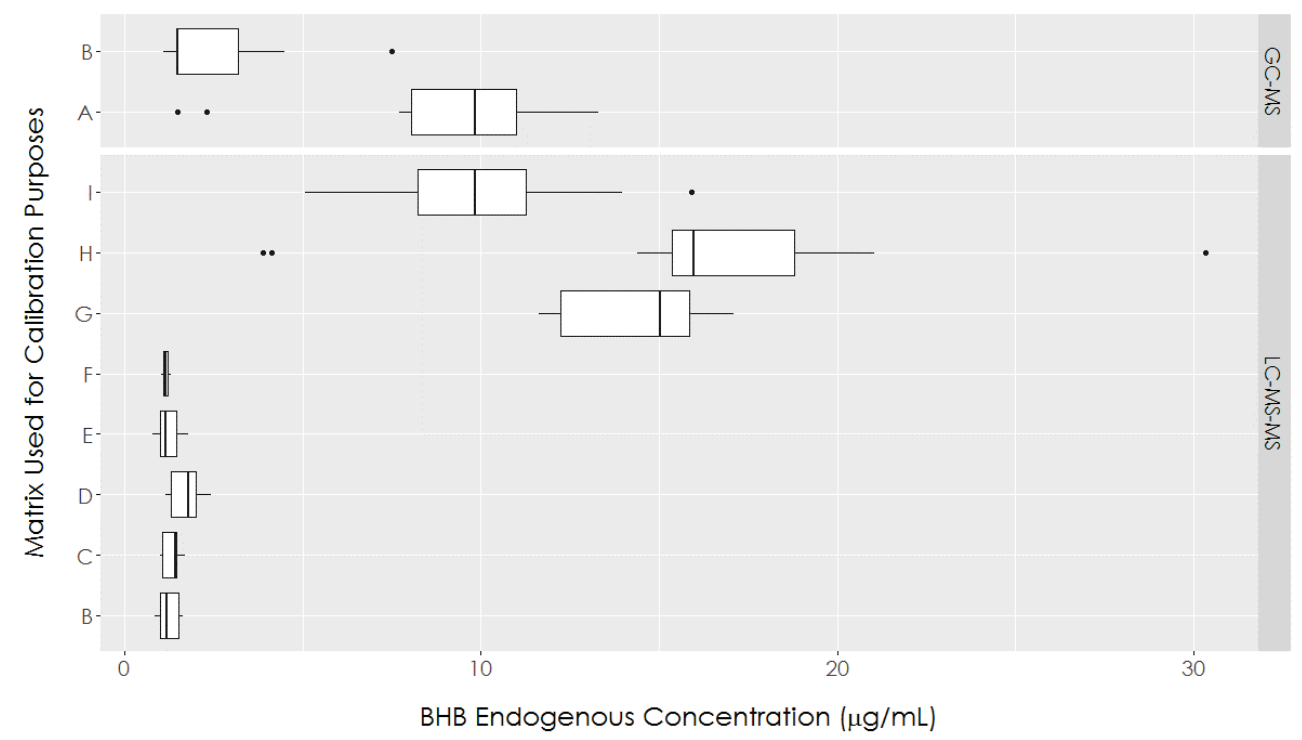

Figure 2: Distribution of the estimated BHB concentrations for each matrix lot. The vertical line within the box is the median value for each lot, while the ends of the box show the upper and lower quartiles. The end point of the lines (whiskers) are the highest and lowest values, excluding the outliers (black dots). This graph is based on all batches analyzed with the different matrix lots (lot $\mathrm{A}, n=10$; lot $\mathrm{B}$ GC-MS, $n=8$; lot B LC-MS-MS, $n=10$; lot C, $n=7$; lot D, $n=8 ;$ lot E, $n=7 ; \operatorname{lot}$ F, $n=6 ; \operatorname{lot} \mathrm{G}$, $n=11 ;$ lot $\mathrm{H}, n=17$; lot I, $n=12$ ).

$11.89 \pm 0.18 \mu \mathrm{g} / \mathrm{mL}$, which supports the conclusion that results within a same matrix lot are consistent.

These results show that, in the case of $\mathrm{BHB}$, endogenous concentrations present in the spiking matrix can be significant. Indeed, at the maximum endogenous concentration measured $(30 \mu \mathrm{g} / \mathrm{mL})$, the endogenous analyte content contributes $15 \%$ of the decision point value $(200 \mu \mathrm{g} / \mathrm{mL})$ to quantifications performed. Applying this correction thus becomes important to uphold accuracy of the results. But regardless of the exact endogenous concentration, performing the correction for the endogenous content will yield a more accurate quantification, at very little cost to productivity, since the $\mathrm{R}$ script developed is run in less than 30 seconds.

\subsection{Additional error folded in by the endogenous concentration correction}

Applying the endogenous concentration correction process will remove the systematic error from the analysis but will necessarily fold in additional indeterminant error. The error on the estimated endogenous concentration $\left(s_{x_{e}}\right)$ must be combined with the error from the estimated uncorrected analyte concentration in the sample $\left(s_{x_{s}}\right)$ to yield the total error for the corrected concentration, $s_{x_{c}}$. The magnitude of the error folded in will depend on a multitude of factors, as demonstrated by equations 7 to 14 . The weighting factor, number of replicate measurements, position of the calibration standards on 
the dynamic range and precision will all play a role in determining the total error and the contribution from the estimated endogenous concentration $\left(s_{x_{e}}\right)$. The multifactorial nature of error magnitude estimation renders instinctual estimation very difficult. In order to help analysts estimate the amount of error involved in the correction procedure, a second automated $\mathrm{R}$ script has been written and is made available to the reader in Supplementary Data 2.

This script has been applied to all 17 GC-MS and 83 LC-MS-MS batches analyzed. For the GC-MS batches, the error added $\left(s_{x_{e}}\right)$ ranges from 0.014 to $0.71 \mu \mathrm{g} / \mathrm{mL}$ (median $0.15 \mu \mathrm{g} / \mathrm{mL}$ ) compared to $6.54 \mu \mathrm{g} / \mathrm{mL}$ as the median error of the uncorrected concentration $\left(s_{x_{s}}\right)$ for the mid-level QC. For LC-MS-MS batches, the error added $\left(s_{x_{e}}\right)$ ranges from 0.0078 to $2.7 \mu \mathrm{g} / \mathrm{mL}$ (median $0.095 \mu \mathrm{g} / \mathrm{mL}$ ) compared to $7.28 \mu \mathrm{g} / \mathrm{mL}$ as the median error of the uncorrected concentration $\left(s_{x_{s}}\right)$. Interestingly, although the absolute error increases after concentration (i.e., $s_{x_{c}}>s_{x_{s}}$ ), the relative (\%) error is reduced by applying the endogenous concentration correction (i.e., $s_{x_{c}} / x_{c}<s_{x_{s}} / x_{s}$ ) in an overwhelming proportion of the cases $(95 \%)$.

At first glance, this was a surprising result, so we decided to confirm its accuracy by running a simulation of the error generated in the calibration and endogenous correction processes. The GC-MS method parameters (calibration standards and QC levels, experimentally observed calibration curve parameters $b_{0}$ and $b_{1}$ ) were used for this procedure. In a simulation such as this one, we know the real value of all parameters $\left(x_{e}, b_{0}, b_{1}\right.$, $\hat{y}_{i}$, etc.) and generate "measured" $y_{i}$ values according to a normal distribution and SWGTOX recommendations (maximum tolerable QC precision of 20\%). The complete $\mathrm{R}$ script is available in Supplementary Data 4. By comparing known and estimated $x_{e}$ values, this in silico evaluation of the endogenous correction process allowed us to confirm that the procedure performs a generally accurate estimation of the endogenous concentration. The histograms of the percentage difference between the calculated and real endogenous concentration, accompanied by the density function, for the different QC levels and weighting schemes, are shown in Figure 3a.

As for the error observed throughout the procedure, the results observed in the in silico simulations match the experimental observations. The absolute error is systematically increased by an amount equal to the estimation error on the endogenous concentration $\left(s_{x_{e}}\right)$. However, the relative (\%) error is almost always reduced by applying the endogenous concentration correction, as shown in Figure 3b. The only situation where this does not happen is with homoscedastic (unweighted) calibrations and lower concentration samples. Not only is the relative error reduced in all other situations, but the higher the concentration of the sample, the greater the drop in relative error. Mathematically, this happens because $s_{x_{c}} / x_{c}$ is smaller than $s_{x_{s}} / x_{s}$. Heteroscedastic ( $1 / x^{2}$ model in particular) will also experience a higher drop in relative error following the correction procedure. In these cases, the correction produces a more accurate concentration with only a marginal increase in absolute error due to the heteroscedasity. Taken together, this improves the relative error. This explains the experimental observations, since the data for both the GC-MS and LC-MS-MS method are heteroscedastic with variance increasing proportionally to the square of the concentration $\left(1 / x^{2}\right.$ model). It is therefore expected to observe mostly a decrease in the relative error following the correction procedure. 

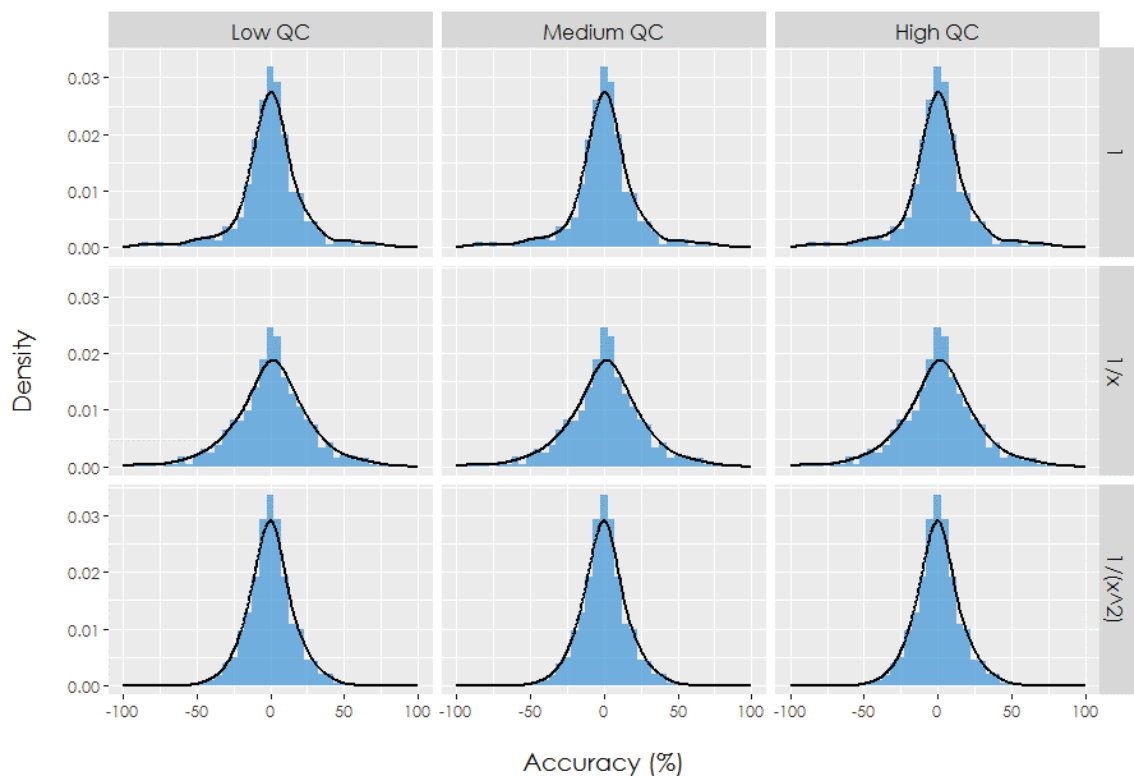

(a) Relative error of the endogenous concentration, calculated as (Calculated Endogenous Concentration - Known Endogenous Concentration) / Known Endogenous Concentration × 100. 1000 simulations per QC level/weight combination have been performed (total 9000 simulations). 1.1\% of data points (outliers) are omitted from this graphical representation for clarity purposes (these mostly have $>100 \%$ relative error). Black lines are density function estimations.

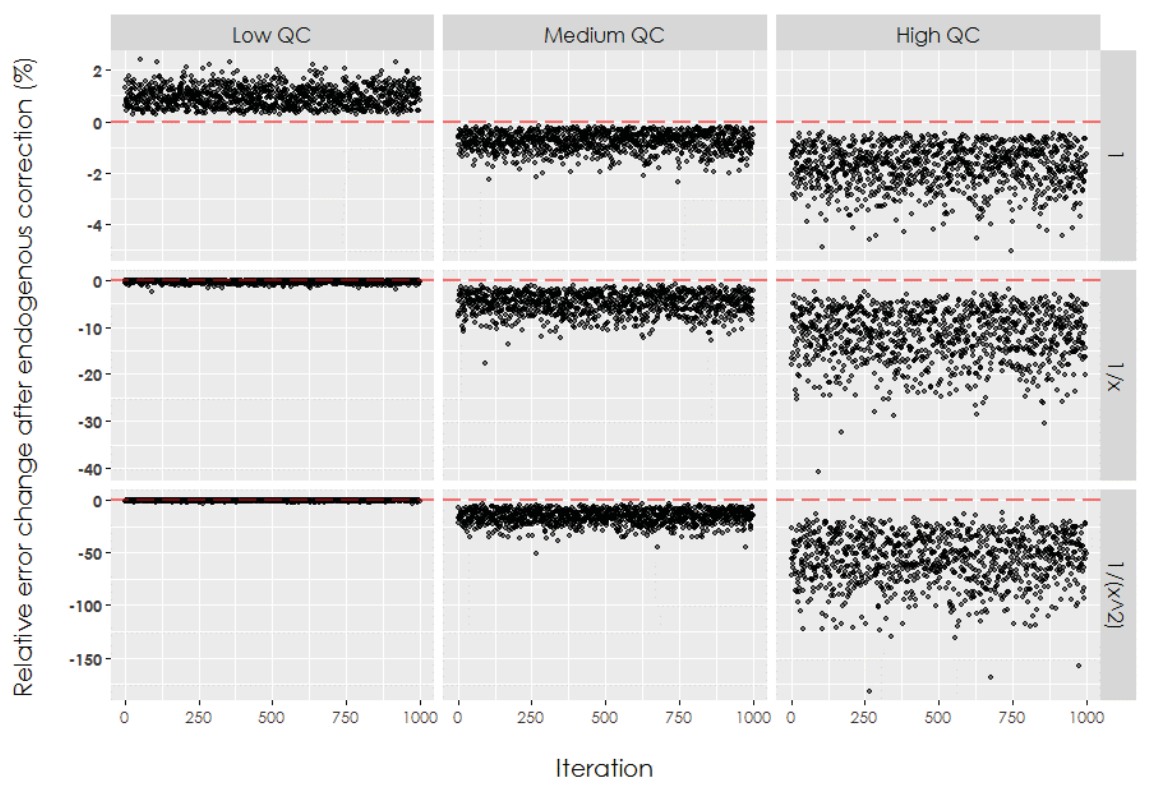

(b) Modeling of the change in the relative error following the endogenous concentration correction process, shown for all QC level/weight combinations. The relative error change is calculated as the relative error on the corrected concentration $\left(s_{x_{s}} / x_{s} \%\right)$ minus the relative error on the uncorrected concentration $\left(s_{x_{c}} / x_{c} \%\right) .1000$ simulations per QC level/weight combination have been performed (total 9000 simulations). Note the different masgitude of the $\mathrm{y}$ axis depending on the weighting scheme studied.

Figure 3: In silico evaluation of the endogenous concentration correction process and estimation of the error. 


\section{Conclusions}

We developed a tool to automatically correct for the presence of endogenous analyte content in matrices used for calibration and QC samples. Instead of using different strategies to more or less sidestep the issue - for example using only standard addition to quantify endogenous analytes, using an analog matrix or analyte, ignoring entirely the endogenous concentration and the bias created in the quantification process - this method can fully take into account the endogenous contribution and correct for it.

This procedure adjusts the expected analyte concentration to match the real, or de facto, concentration present in the calibration standard, i.e., the amount of analyte added plus the endogenous amount. The endogenous concentration is estimated via the calibration standards by treating them as a standard-addition experiment/calibration. Although there are several different ways to perform such a correction mathematically, adjusting the expected concentration allows seamless integration of the correction procedure with production operations performed in commercial data analysis software such as Mass Hunter or MultiQuant.

We have described in detail how the correction procedure can be applied in a production setting to correct for the endogenous concentration present in the spiking matrix. In validation procedures, addressing the endogenous concentration problem head-on will require somewhat more work. The endogenous concentration will need to be determined for every matrix used in the validation procedures, and results corrected accordingly. The endogenous concentration for each matrix can be determined with the tool developed here. Ultimately, this supplementary work at validation time, combined with the endogenous concentration correction during the production operations, will yield an unbiased and more reliable quantification of the analyte for the long term.

The necessity to perform such a correction will vary based on the targeted application and the endogenous concentration of the analyte. However, at any detectable endogenous presence, correcting for the presence of analyte in the spiking matrix will generate a more accurate quantification.

We have applied this correction procedure in a production setting to two methods used for BHB quantification; one GC-MS and one LC-MS-MS method. Endogenous BHB concentrations ranging from 0 to $30 \mu \mathrm{g} / \mathrm{mL}$ have been found in whole human blood matrices. Correcting for this content prevents a bias of up to $15 \%$ of the BHB decision point for hyperglycemia $(200 \mu \mathrm{g} / \mathrm{mL})$. It can therefore be important to correct for endogenous concentrations, which can be significant.

This procedure does increase the estimation error on the concentration for quantified unknowns. The exact magnitude of the error added by this process very much depends on specific method parameters, such as the number and distribution of calibration standards, the number of replicate measurement, homo or heteroscedasticity of the data, etc. 
We have developed an $\mathrm{R}$ script (Supplementary Data 2) which allows users to get an estimation of the error added with their specific method. For the BHB quantification methods presented in this paper, the additional error folded in by the correction procedure amounts to a few percent $(<3 \%)$ at most. The small error added by the procedure, especially when compared to the potential for removing systematic bias makes this an attractive solution to deal with endogenous analytes in validation and production settings.

\section{Acknowledgements}

The authors wish to thank Cynthia Côté for her questions and ideas.

\section{References}

[1] Scientific Working Group for Forensic Toxicology, Scientific Working Group for Forensic Toxicology (SWGTOX) Standard Practices for Method Validation in Forensic Toxicology, Journal of Analytical Toxicology 37 (2013) 452-474.

[2] Pirro, Valentina and Valente, Valeria and Oliveri, Paolo and De Bernardis, Angela and Salomone, Alberto and Vincenti, Marco, Chemometric evaluation of nine alcohol biomarkers in a large population of clinically-classified subjects: pre-eminence of ethyl glucuronide concentration in hair for confirmatory classification, Analytical and Bioanalytical Chemistry 401 (2011) 2153.

[3] Fulgoni III, Victor L and Keast, Debra R and Lieberman, Harris R, Trends in intake and sources of caffeine in the diets of US adults: 2001-2010, The American Journal of Clinical Nutrition 101 (2015) 1081-1087.

[4] Andresen-Streichert, Hilke and Müller, Alexander and Glahn, Alexander and Skopp, Gisela and Sterneck, Martina, Alcohol biomarkers in clinical and forensic contexts, Deutsches Ärzteblatt International 115 (2018) 309.

[5] N. C. for Health Statistics, National health and nutrition examination survey. hyattsville, md: U.s. department of health and human services, centers for disease control and prevention, 2018.

[6] Elliott, Simon and Smith, Christopher and Cassidy, Diane, The post-mortem relationship between beta-hydroxybutyrate (BHB), acetone and ethanol in ketoacidosis, Forensic Science International 198 (2010) 53-57.

[7] Osuna, Eduardo and Vivero, Guillermo and Conejero, Josefa and Abenza, José M and Martínez, Pedro and Luna, Aurelio and Pérez-Cárceles, María D, Postmortem vitreous humor $\beta$ hydroxybutyrate: its utility for the postmortem interpretation of diabetes mellitus, Forensic Science International 153 (2005) 189-195.

[8] Hess, Cornelius and Sydow, Konrad and Kueting, Theresa and Kraemer, Michael and Maas, Alexandra, Considerations regarding the validation of chromatographic mass spectrometric methods for the quantification of endogenous substances in forensics, Forensic Science International 283 (2018) $150-155$.

[9] Wille, Sarah MR and Peters, Frank T and Di Fazio, Vincent and Samyn, Nele, Practical aspects concerning validation and quality control for forensic and clinical bioanalytical quantitative methods, Accreditation and Quality Assurance 16 (2011) 279.

[10] Skoog, Douglas A., Holler, F. James, Crouch, Stanley R., Selecting an Analytical Method, in Principles of Instrumental Analysis, Cengage Learning, Belmont, United States, 6 edition, 2006, pp. 17-21.

[11] Elliott, Simon P, Gamma hydroxybutyric acid (GHB) concentrations in humans and factors affecting endogenous production, Forensic Science International 133 (2003) 9-16.

[12] Jemal, Mohammed and Schuster, Alan and Whigan, Daisy B, Liquid chromatography/tandem mass spectrometry methods for quantitation of mevalonic acid in human plasma and urine: method validation, demonstration of using a surrogate analyte, and demonstration of unacceptable matrix effect in spite of use of a stable isotope analog internal standard, Rapid Communications in Mass Spectrometry 17 (2003) 1723-1734. 
[13] Ji, Dajeong and Jang, Choon-Gon and Lee, Sooyeun, A sensitive and accurate quantitative method to determine $\mathrm{N}$-arachidonoyldopamine and N-oleoyldopamine in the mouse striatum using columnswitching LC-MS-MS: use of a surrogate matrix to quantify endogenous compounds, Analytical and Bioanalytical Chemistry 406 (2014) 4491-4499.

[14] Jones, Barry R and Schultz, Gary A and Eckstein, James A and Ackermann, Bradley L, Surrogate matrix and surrogate analyte approaches for definitive quantitation of endogenous biomolecules, Bioanalysis 4 (2012) 2343-2356.

[15] Binz, Tina M and Braun, Ueli and Baumgartner, Markus R and Kraemer, Thomas, Development of an LC-MS/MS method for the determination of endogenous cortisol in hair using 13C3-labeled cortisol as surrogate analyte, Journal of Chromatography B 1033 (2016) 65-72.

[16] Kang, Soyoung and Oh, Seung Min and Chung, Kyu Hyuck and Lee, Sooyeun, A surrogate analytebased LC-MS/MS method for the determination of $\gamma$-hydroxybutyrate (GHB) in human urine and variation of endogenous urinary concentrations of GHB, Journal of Pharmaceutical and Biomedical Analysis 98 (2014) 193-200.

[17] Savard, Stéphanie and Lapointe, Christine and Lamarche, Martine and Mireault, Pascal, Development of a new method for simultaneous quantitative BHB and GHB analysis by GC-MS, Standard, Poster presented at the International Association of Forensic Sciences (IAFS) 2017 Meeting, Toronto, Canada, 2017.

[18] Thermo Scientific, Instructions.: BSTFA + TMCS N,O-bis(Trimethylsilyl)trifluoroacetamide with Trimethylchlorosilane, Standard, https://fscimage.fishersci.com/images/D00369 .pdf (Retrieved January 28th 2018), 2017.

[19] ISO/IEC 17025:2005, General requirements for the competence of testing and calibration laboratories, Standard, International Organization for Standardization, Geneva, Switzerland, 2005.

[20] CAN-P-1578, Guidelines for the Accreditation of Forensic Testing Laboratories, Standard, Standards Council of Canada (Conseil canadien des normes), Ottawa, Canada, 2009.

[21] Desharnais, Brigitte and Camirand-Lemyre, Félix and Mireault, Pascal and Skinner, Cameron D, Procedure for the Selection and Validation of a Calibration Model I - Description and Application, Journal of Analytical Toxicology 41 (2017) 261-268.

[22] Desharnais, Brigitte and Camirand-Lemyre, Félix and Mireault, Pascal and Skinner, Cameron D Procedure for the Selection and Validation of a Calibration Model II — Theoretical Basis, Journal of Analytical Toxicology 41 (2017) 269-276.

[23] D.L. Massart, B.G.M. Vandeginste, L.M.C Buydens, S. De Jong, P.J. Lewi, J. Smeyers-Verbeke, Straight Line Regression and Calibration, in: Handbook of Chemometrics and Qualimetrics: Part A, volume 20A of Data Handling in Science and Technology, Elsevier, Amsterdam, Netherlands, 1997, pp. 171-230.

[24] Navidi, William, Propagation of Error, in: Statistics for Engineers and Scientists, Mc Graw Hill Education, New York, United States, 2014, pp. 164-199.

[25] Zörntlein, SW and Kopp, A and Becker, Julie and Kaufmann, TJ and Röhrich, J and Urban, R, In vitro production of GHB in blood and serum samples under various storage conditions, Forensic Science International 214 (2012) 113-117. 